\title{
EVENTUAL DISCONJUGACY OF SELFADJOINT FOURTH ORDER LINEAR DIFFERENTIAL EQUATIONS
}

\author{
G. B. GUSTAFSON
}

\begin{abstract}
At the 1969 Differential Equations Conference held at Knoxville, Tennessee, $Z$. Nehari asked the following question about fourth-order selfadjoint linear differential equations: "Assume that solutions of the equation have only a finite number of zeros on $t \geqq A$. Does there exist a half-line on which no solution of the equation has more than three zeros?"' In this paper sufficient conditions are given for the equation $\left(p(t) y^{\prime \prime}\right)+\left(q(t) y^{\prime}\right)^{\prime}=0$ to have the property that solutions possess only a finite number of zeros. This theorem is then used to construct an example which answers the above question in the negative. The example also shows that if on each half-line the equation has a solution with two consecutive double zeros, then it need not follow that there is a solution with infinitely many zeros.
\end{abstract}

1. Introduction. The general fourth-order selfadjoint linear differential equation

$$
\left(p y^{\prime \prime}\right)^{\prime \prime}+\left(q y^{\prime}\right)^{\prime}+r y=0, \quad p, q, r \text { in } C[A, \infty), p>0,
$$

has received considerable attention since the appearance of Leighton and Nehari's fundamental paper [6] in 1958. If some nontrivial solution of (1.1) has an infinity of zeros, then (1.1) is called oscillatory; in the contrary case (1.1) is called nonoscillatory. The equation is called disconjugate on a set $E$ provided the number of zeros of a nontrivial solution is less than the order of the equation; eventual disconjugacy is disconjugacy on some halfline.

A substantial percentage of known oscillation theorems use the following scheme: nonoscillation $\rightarrow$ eventual disconjugacy $\rightarrow$ a coefficient condition. The defect in this scheme for higher order equations is the first implication: it only holds for special cases; see [2].

The question of the equivalence of nonoscillation and eventual disconjugacy makes sense for all linear differential equations, but the selfadjoint case is the most logical generalization of the Sturm theory; hopefully, this equivalence should generalize to selfadjoint equations.

Received by the editors August 15, 1969 and, in revised form, January 7, 1972.

AMS 1969 subject classifications. Primary 3442; Secondary 3430, 3450.

Key words and phrases. Oscillation, nonoscillation, disconjugacy, eventually disconjugate, disconjugate in the sense of Reid, $p$ th conjugate point. 
It does indeed generalize to third-order equations, but it fails for fourth-order selfadjoint equations, as will be seen below.

The purpose of this paper is to answer in the negative the following: Assume that each solution of (1.1) has only a finite number of zeros on $t \geqq A$. Does there exist a half-line on which no solution of (1.1) has more than three zeros?

Suppose that on each half-line equation (1.1) has a solution with two consecutive double zeros. Does (1.1) have a solution with infinitely many zeros?

The $p$ th conjugate point belonging to $t=a$ is denoted by $\eta_{p}(a)$, and equals the minimum of the set of all $b>a$ for which the equation (1.1) has a solution with a zero at $t=a$ and $p+3$ zeros on $a \leqq t \leqq b$, counting multiplicities. In [10] it is proved that this minimum exists provided there exists a solution $y(t) \not \equiv 0$ that vanishes at $t=a$ and has at least $p+3$ zeros on $t \geqq a$, counting multiplicities.

2. THEOREM. The equation $\left(p y^{\prime \prime}\right)^{\prime \prime}+\left(q y^{\prime}\right)^{\prime}=0$ is nonoscillatory on $t \geqq A$ if $\left(p y^{\prime \prime}\right)^{\prime}+q y^{\prime}=0$ has two solutions $U$ and $V$ such that

(i) $\lim _{t \rightarrow \infty} U(t)=\infty, \lim _{t \rightarrow \infty} V(t)=\infty$,

(ii) $\lim _{t \rightarrow \infty}(U(t) / V(t))=0$,

(iii) $\int_{A}^{t} U^{\prime}(s) V(s) d s / V(t)=O(1)$.

Proof. The function $w(t)=-U(t) V(t)+2 \int_{A}^{t} U^{\prime}(s) V(s) d s$ is a solution of the fourth-order equation independent from $1, U$, and $V$. If

$$
y(t)=C_{1}+C_{2} U(t)+C_{3} V(t)+C_{4} \cdots(t), \quad t \geqq A,
$$

vanishes infinitely often on $t \geqq A$, then the equation

$$
0=\frac{C_{1}}{V(t)}+C_{2} \frac{U(t)}{V(t)}+C_{3}-C_{4} U(t)+2 C_{4}\left(\int_{A}^{t} U^{\prime}(s) V(s) d s\right) / V(t)
$$

has a solution $t$ on every half-line. The right side of this equation is dominated by $-C_{4} U(t)$ for $C_{4} \neq 0$; therefore, $C_{4}=0$. Letting $t \rightarrow \infty$ and applying (ii) gives $C_{3}=0$. Then $C_{1}+C_{2} U(t)=0$ has a solution $t$ in every half-line; by (i), $C_{2}=0$, and finally $C_{1}=0$. Hence $y \equiv 0$, and the equation is nonoscillatory on $t \geqq A$.

REMARK. The referee has kindly pointed out that $O(1)$ in (iii) can be replaced by $o\left(\frac{1}{2} U(t)\right)$.

3. A nonoscillatory equation (1.1) that is not eventually disconjugate. Let $\left(p z^{\prime}\right)^{\prime}+q z=0$ be the equation (see [3]) whose fundamental solutions are

$$
u=1 / t+\sin t, \quad v=\varepsilon+\cos t .
$$


The Wronskian of $v$ and $u$ is $W(v, u)=1+\varepsilon \cos t-\left(1 / t^{2}\right)(\varepsilon+\cos t)+$ $(1 / t) \sin t$; so there is a number $A>0$ and a number $\varepsilon>0$ such that $W(v, u)>$ 0 for $t \geqq A$. Therefore, $\left(p z^{\prime}\right)^{\prime}+q z=0$ is a nonsingular linear differential equation on $t \geqq A$ with $C^{\infty}$ coefficients.

Define $U(t)=\log t-\cos t, V(t)=\varepsilon t+\sin t$. Then $U^{\prime}=u$, and $V^{\prime}=v$. Hence, 1, $U, V$ form a fundamental set of solutions for the nonsingular third order linear differential equation $\left(p y^{\prime \prime}\right)^{\prime}+q y^{\prime}=0$. Furthermore,

$$
\lim _{t \rightarrow \infty} U(t)=\infty, \quad \lim _{t \rightarrow \infty} V(t)=\infty, \quad \text { and } \quad \lim _{t \rightarrow \infty} \frac{U(t)}{V(t)}=0 .
$$

In addition,

$$
\int_{A}^{t} U^{\prime}(s) V(s) d s / V(t)=\frac{\left(\varepsilon+\frac{1}{2}-\varepsilon \cos t\right) t+O(1)}{\varepsilon t+\sin t}=O(1) .
$$

Therefore, (i)-(iii) of Theorem 2 hold. The equation $\left(p y^{\prime \prime}\right)^{\prime \prime}+\left(q y^{\prime}\right)^{\prime}=0$ will be modified on a "small" set to obtain the example.

Let $\left[a_{0}, b_{0}\right],\left[a_{1}, b_{1}\right],\left[a_{2}, b_{2}\right], \cdots$ be disjoint intervals in $[A, \infty)$ such that $a_{0}=A, b_{n}<a_{n+1}, n \geqq 0, \lim _{n \rightarrow \infty} a_{n}=\infty$, and $\sum_{n=0}^{\infty} \int_{a_{n}}^{b_{n}} t d t<\infty$.

Put $J_{n}=\left[a_{n}, b_{n}\right], n \geqq 0$, and let $J$ equal the union of all the $J_{n}$ 's. Suppose it is possible to find $C^{4}$ functions $u^{*}(t), v^{*}(t)$ which are fundamental solutions of a second order equation $\left(p^{*} z^{\prime}\right)^{\prime}+q^{*} z=0$ on $t \geqq A$ such that $u^{*}=u$ and $v^{*}=v$ off $J,\left|u^{*}\right| \leqq 1+|u|,\left|v^{*}\right| \leqq 1+|v|$ on $J$,

$$
u^{*}(t)=\cos \frac{n \pi\left(t-c_{n}\right)}{\left(d_{n}-c_{n}\right)}, \quad v^{*}(t)=\sin \frac{n \pi\left(t-c_{n}\right)}{\left(d_{n}-c_{n}\right)},
$$

on an interval $\left[c_{n}, d_{n}\right] \subset\left(a_{n}, b_{n}\right), n \geqq 1$. It will be shown that $\left(p^{*} y^{\prime \prime}\right)^{\prime \prime}+$ $\left(q^{*} y^{\prime}\right)^{\prime}=0$ is nonoscillatory on $t \geqq A$ and every number $a \geqq A$ has a $p$ th conjugate point, $p \geqq 1$.

For this purpose, define

$$
U^{*}(t)=U(t)+\int_{A}^{t}\left(u^{*}(s)-u(s)\right) d s
$$

and

$$
V^{*}(t)=V(t)+\int_{-1}^{t}\left(v^{*}(s)-v(s)\right) d s
$$

Then surely $1, V^{*}, U^{*}$ are linearly independent solutions of $\left(p^{*} y^{\prime \prime}\right)^{\prime \prime}+$ $\left(q^{*} y^{\prime}\right)^{\prime}=0$. It will be demonstrated that $U^{*}$ and $l^{*}$ satisfy (i)-(iii) of Theorem 2.

Because $v^{*}=v$ and $u^{*}=u$ off $J, V^{*}=V+O(1)$ and $L^{*}=U+O(1)$. Therefore, (i) holds. Since $U^{*}=O(\log t)$, (ii) holds. To establish (iii), 
calculate

$$
\begin{aligned}
\int_{A}^{t} U^{* \prime}(s) V^{*}(s) d s / V^{*}(t)= & \int_{A}^{t} u^{*}(s) V^{*}(s) d s / V^{*}(t) \\
= & \int_{A}^{t}\left(u^{*}(s)-u(s)\right) V^{*}(s) d s / V^{*}(t) \\
& +\int_{A}^{t} u(s) V(s) d s / V^{*}(t) \\
& +\int_{A}^{t} u(s)\left(V^{*}(s)-V(s)\right) d s / V^{*}(t) \\
= & \int_{J} O(1) O(s) d s / V^{*}(t)+\frac{O(t)}{V^{*}(t)} \\
& +\int_{A}^{t} O(1) d s / V^{*}(t)=\dot{O}(1) .
\end{aligned}
$$

Therefore, $U^{*}$ and $V^{*}$ satisfy (i)-(iii) of Theorem 2. Accordingly, the equation $\left(p^{*} y^{\prime \prime}\right)^{\prime \prime}+\left(q^{*} y^{\prime}\right)^{\prime}=0$ is nonoscillatory on $t \geqq A$.

Suppose $a \in[A, \infty)$. It will be shown that each $p$ th conjugate point $\eta_{p}(a)$ exists, $p \geqq 1$. Given an integer $p \geqq 1$, select an integer $n$ such that $a_{n}>a, n>p+3$. Define $k(t)=n \pi\left(t-c_{n}\right) /\left(d_{n}-c_{n}\right)$ and suppose that $U^{*}(t)=$ $h_{1} \sin k(t)+C_{1}, V^{*}(t)=h_{2} \cos k(t)+C_{2}$, on $c_{n} \leqq t \leqq d_{n}$. Select constants $m_{1}$ and $m_{2}$ not both zero such that $m_{1}\left[U^{*}(a)-C_{1}\right]+m_{2}\left[V^{*}(a)-C_{2}\right]=0$. Put $y(t)=m_{1} U^{*}(t)+m_{2} V^{*}(t)-m_{1} C_{1}-m_{2} C_{2}$. Then $y(t)$ is a nontrivial solution of $\left(p^{*} y^{\prime \prime}\right)^{\prime \prime}+\left(q^{*} y^{\prime}\right)^{\prime}=0, y(a)=0$, and $y(t)=m_{1} h_{1} \sin k(t)+m_{2} h_{2} \cos k(t)$ on $c_{n} \leqq t \leqq d_{n}$. Because $v(t)$ has at least $p+3$ zeros on the interval $a \leqq t \leqq d_{n}$, a standard theorem on conjugate points (see [4], [10]) shows that $\eta_{p}(a)$ exists. Furthermore, a constant can be added to $y(t)$ so that the solution so obtained has $p+3$ double zeros on $c_{n} \leqq t \leqq d_{n}$.

The equation $\left(p y^{\prime \prime}\right)^{\prime \prime}+\left(q y^{\prime}\right)^{\prime}=0$ is nonoscillatory, but not eventually disconjugate. Furthermore, given any half-line $t \geqq T$, and any integer $N>0$, there is a solution of the equation with $N$ consecutive double zeros. Therefore, the equation is not disconjugate in the sense of Reid (see [9], [10]).

To establish the existence of the special functions $u^{*}$ and $v^{*}$, the following lemmas will be used:

4. Lemma. Let $x(t)$ and $y(t)$ be solutions of $\left(p z^{\prime}\right)^{\prime}+q z=0$ such that $x(T)=0, x^{\prime}(T)>0, y(T)>0$, and suppose $G(t)$ is the solution of

$$
G^{\prime}(t)=\frac{y(T) x^{\prime}(T)}{p(t) f^{2}(t)}, \quad G(T)=2 k \pi,
$$


where $F^{2}(t)=y^{2}(t)+x^{2}(t)$, and $k$ is an integer. Then $x(t)=F(t) \sin G(t)$ and $y(t)=F(t) \cos G(t)$ for $t \geqq T$.

5. LemMa. Given an integer $N>0$, there exists an $r(t)$ in $C^{-1}[c, d]$ such that

(i) $r(c)=0, r(d)=1$,

(ii) $r^{\prime}(t)>0$ on $(c, d)$,

(iii) $r^{\prime}(t)$ has a zero of order $N-1$ at $t=c$ and $t=d$ (see [4]).

6. Lemma. Suppose $E$ is connected, $[c, d] \subset E, f_{1}, f_{2}, g_{1}, g_{2} \in C^{N}(E)$, $f_{1}^{2}(t) g_{1}^{\prime}(t)>0, f_{2}^{2}(t) g_{2}^{\prime}(t)>0$ on $E$. There exist functions $f, g \subseteq C^{N}(E)$ such that

(i) $f=f_{1}$ on $(-\infty, c] \cap E, f=f_{2}$ on $[d, \infty) \cap E$,

(ii) $g=g_{1}$ (modulo $\left.2 \pi\right)$ on $(-\infty, c] \cap E, g=g_{2}(\operatorname{modulo} 2 \pi)$ on $[d, \infty) \cap E$,

(iii) $g^{\prime}(t) \geqq \min \left\{g_{1}^{\prime}(t), g_{2}^{\prime}(t)\right\}$ on $c \leqq t \leqq d$,

(iv) $\min \left\{f_{1}(t), f_{2}(t)\right\} \leqq f(t) \leqq \max \left\{f_{1}(t), f_{2}(t)\right\}$ on $c \leqq t \leqq d$.

Proof of Lemma 6. Let $r(t)$ be the function of Lemma 5. Select an integer $k>0$ such that $g_{1}(d) \leqq g_{2}(c)+2 k \pi$. Define

$$
\begin{aligned}
& f(t)=f_{1}(t), \\
& t \in(-\infty, c] \cap E, \\
& =r(t) f_{2}(t)+(1-r(t)) f_{1}(t), \\
& c \leqq t \leqq d, \\
& =f_{2}(t), \\
& t \in[d, \infty) \cap E, \\
& g(t)=g_{1}(t) \text {, } \\
& t \in(-\infty, c] \cap E, \\
& =r(t)\left(g_{2}(t)+2 k \pi\right)+(1-r(t)) g_{1}(t), \\
& c \leqq t \leqq d \text {, } \\
& =g_{2}(t)+2 k \pi \text {, } \\
& t \in[d, \infty) \cap E \text {. }
\end{aligned}
$$

Then (i) and (ii) hold. On $c \leqq t \leqq d$,

$$
\begin{aligned}
f(t) & =r(t) f_{2}(t)+(1-r(t)) f_{1}(t) \\
& \leqq r(t) \max \left\{f_{1}(t), f_{2}(t)\right\}+(1-r(t)) \max \left\{f_{1}(t), f_{2}(t)\right\} \\
& =\max \left\{f_{1}(t), f_{2}(t)\right\} ;
\end{aligned}
$$

likewise, $f(t) \geqq \min \left\{f_{1}(t), f_{2}(t)\right\}$. This demonstrates (iv). To prove (iii), compute

$$
g^{\prime}(t)=r^{\prime}(t)\left(g_{2}(t)+2 k \pi-g_{1}(t)\right)+r(t) g_{2}^{\prime}(t)+(1-r(t)) g_{1}^{\prime}(t) .
$$

Since $g_{1}$ and $g_{2}$ are increasing,

$$
g_{1}(t) \leqq g_{1}(d) \leqq g_{2}(c)+2 k \pi \leqq g_{2}(t)+2 k \pi
$$

for $c \leqq t \leqq d$. By (ii) of Lemma 5 , the above formulas give

$$
g^{\prime}(t) \geqq r(t) g_{2}^{\prime}(t)+(1-r(t)) g_{1}^{\prime}(t) \geqq \min \left\{g_{1}^{\prime}(t), g_{2}^{\prime}(t)\right\} .
$$

The proof is complete.

The construction of $u^{*}$ and $v^{*}$ will now proceed. Define $u^{*}=u$ and $v^{*}=$ $v$ off the set $\bigcup_{n=1}^{\infty}\left(a_{n}, b_{n}\right)$. The definition of $u^{*}$ and $\iota^{*}$ will be completed 
by defining these functions on each $J_{n}, n \geqq 1$. Let $\left[c_{n}, d_{n}\right] \subset\left(a_{n}, b_{n}\right)$, and put $E=\left[b_{n-1}, b_{n}\right],[c, d]=\left[a_{n}, c_{n}\right]$ in Lemma 6. Write $u(t)=f_{1}(t) \cos g_{1}(t)$, $v^{\prime}(t)=f_{1}(t) \sin g_{1}(t)$ via Lemma 4 , and put $f_{2}(t)=1, g_{2}(t)=n \pi\left(t-c_{n}\right) /\left(d_{n}-c_{n}\right)$. Apply Lemma 6 to find functions $f(t)$ and $g(t)$ as asserted in the lemma, and put $u^{*}(t)=f(t) \cos g(t), v^{*}(t)=f(t) \sin g(t)$ on $\left[a_{n}, d_{n}\right]$. Similarly, $u^{*}$ and $v^{*}$ can be defined on $\left[d_{n}, b_{n}\right]$. The functions $u^{*}$ and $v^{*}$ resulting from this construction satisfy

$$
W\left(u^{*}(t), v^{*}(t)\right)>0, \quad\left|u^{*}(t)\right| \leqq|u(t)|+1, \quad\left|v^{*}(t)\right| \leqq|v(t)|+1,
$$

on $\left[b_{n-1}, a_{n+1}\right], n \geqq 1$, by Lemma 6 . The existence of the functions $u^{*}$ and $v^{*}$ is now established.

The example above shows that selfadjoint equations need not inherit disconjugacy from nonoscillation criteria. Moreover, conjugate point information is not always sufficient to determine asymptotic properties such as oscillation and nonoscillation.

For positive results see [1], [2], [6], [7], [8], [9], [11]. Some unsolved problems appear in [1], [2], [7].

\section{REFERENCES}

1. J. H. Barrett, Sistems-disconjugacy of a fourth-order differential equation, Proc. Amer. Math. Soc. 12 (1961), 205-213. MR 24 \#A304.

2. - - Oscillation theory of ordinary differential equations, Associated Western Universities Differential Equations Sympos., Boulder, Colorado, Summer 1967.

3. J. M. Dolan, On the relationship between the oscillatory behavior of a linear thirdorder differential equation and its adjoint, J. Differential Equations 7 (1970), 367-388. MR 41 \#568.

4. G. Gustafison, Conjugate point properties for nth order linear differential equations Ph.D. Dissertation, Arizona State University, Tempe, Ariz., 1968.

5. P. Hartman, Ordinary differential equations, Wiley, New York, 1964. MR 30 \#1270.

6. W. Leighton and Z. Nehari, On the oscillation of self-adjoint linear differential equations of the fourth order, Trans. Amer. Math. Soc. 89 (1958), 325-377. MR 21 \#1429.

7. Z. Nehari, Non-oscillation criteria for $n$th order linear differential equations, Duke Math. J. 32 (1965), 607-615. MR 32 \#4338.

8. -_- Disconjugate linear differential operators. Trans. Amer. Math. Soc. 129 (1967), 500-516. MR 36 \#2860.

9. W. T. Reid, Oscillation criteria for self-adjoint differential systems, Trans. Amer. Math. Soc. 101 (1961), 91 106. MR 24 \#A3349.

10. T. L. Sherman, Propertic's of solutions of nth order linear differential equations, Pacific J. Math. 15 (1965), 1045-1060. MR 32 \#2654.

11. M. E. Švec, Sur une propriété des intégrales de l'équation $y^{(n)}+Q(X) y=0, n=3$, 4, Czechoslovak Math. J. 7 (82) (1957), 450-462. MR 20 \#1816.

Department of Maihmatics, University of Utah, Salt lake City, Utah 84112 